\title{
Compressive holography with a single-pixel detector
}

\author{
Pere Clemente, ${ }^{1,2}$ Vicente Durán, ${ }^{1}$ Enrique Tajahuerce, ${ }^{1, *}$ Pedro Andrés, ${ }^{3}$ Vicent Climent, ${ }^{1}$ and Jesús Lancis ${ }^{1}$ \\ ${ }^{1}$ GROC UJI, Institut de Noves Tecnologies de la Imatge (INIT), Universitat Jaume I, Castelló E12071, Spain \\ ${ }^{2}$ Servei Central d'Instrumentació, Universitat Jaume I, Castelló E12071, Spain \\ ${ }^{3}$ Departament d'Òptica, Universitat de València, Burjassot E46100, Spain \\ ${ }^{*}$ Corresponding author: enrique.tajahuerce@uji.es
}

Received May 3, 2013; accepted June 3, 2013;

posted June 18, 2013 (Doc. ID 190026); published July 10, 2013

\begin{abstract}
This Letter develops a framework for digital holography at optical wavelengths by merging phase-shifting interferometry with single-pixel optical imaging based on compressive sensing. The field diffracted by an input object is sampled by Hadamard patterns with a liquid crystal spatial light modulator. The concept of a single-pixel camera is then adapted to perform interferometric imaging of the sampled diffraction pattern by using a Mach-Zehnder interferometer. Phase-shifting techniques together with the application of a backward light propagation algorithm allow the complex amplitude of the object under scrutiny to be resolved. A proof-of-concept experiment evaluating the phase distribution of an ophthalmic lens with compressive phase-shifting holography is provided. @ 2013 Optical Society of America

OCIS codes: (100.3175) Interferometric imaging; (090.1760) Computer holography; (110.1758) Computational imaging.

http://dx.doi.org/10.1364/OL.38.002524
\end{abstract}

Digital holography records and recovers numerically the complex amplitude distribution of diffraction patterns. Therefore, it provides an effective phase imaging technique, which has become an invaluable tool in biomedical imaging at optical wavelengths [1]. In brief, digital holography works by interfering a coherent signal beam, the field diffracted by the phase object, with a reference beam. The resulting hologram is recorded onto a CCD or CMOS detector. The phase information is subsequently recovered via digital back propagation of the hologram to the object plane using a diffraction-based algorithm. A unique advantage of the digital recording is that it permits the application of a vast array of digital processing techniques and numerical algorithms to compensate, for instance, optical aberrations. In phase-shifting holography, the hologram results from a limited set of interferograms each adding a constant phase delay between the signal and the reference beam [2]. Parallel capturing of the phase-shifted holograms has been shown [주느. Data compression techniques have also been applied for image reconstruction only by phase data [5].

In a different context, the use of compressive sensing (CS) techniques is revolutionizing many different areas in electrical engineering, and imaging is not an exception. Most images of interest that are obtained in experiments or by numerical computation of natural phenomena are sparse, i.e., they contain many zero coefficients when transformed into an appropriate basis (e.g., the discrete cosine transform). In CS, it is possible to make use of this fact to measure a sparsely represented $N$-dimensional image from $M<N$ incoherent measurements using an optimization algorithm [6,7]. Note that the number of measurements would be deemed insufficient by use of conventional imaging techniques. The approach is so named because it allows image compression to be performed during measurement rather than in the postprocessing stage. Although sparsity is assumed, it is not known prior to the measurement what coefficients have appreciable amplitude. CS must determine the elements that are significant, as well as find their values.

The quintessential example of CS is the single-pixel camera $[\underline{8}, \underline{9}]$. The object information is obtained by imaging the scene throughout structured masks implemented with the aid of a programmable spatial light modulator (SLM). A photodiode (single-pixel detector) measures the throughput as the pattern generated in the SLM changes in a sequential manner. In our approach the object is sampled by Hadamard patterns [10]. The benefit of Hadamard sampling is that it more efficiently uses the available energy flux (multiplex advantage). In other words, for pixelated imaging, the light flux is distributed over the $N$ pixels of the image and, subsequently, scales inversely with $N$. On the contrary, the average flux per each Hadamard pattern is independent of the number of pixels, and in fact each measurement collects on average half of the total flux. Further, for incoherent compressive measurements, the sub-Nyquist image sampling overcomes the fundamental trade-off between speed and resolution, as the acquisition time is reduced. In the context of holography, it should be mentioned that compressive algorithms have been used although in a pixelated detection scheme only [11,12].

By leveraging phase-shifting digital holography and CS, in this Letter we present imaging of phase objects by single-pixel digital holography. The key point is to formulate the measurement problem in terms of the complex amplitude transmittance rather than using the intensity, which is the variable of choice for conventional CS-based single-pixel imaging. The above proposition constitutes the generalization of our previously reported ghost holography technique [13]. Experimental results are validated by retrieving the phase map corresponding to a conventional ophthalmic lens.

The scheme of the device is shown in Fig. 1. It consists of a Mach-Zehnder interferometer that includes a phase shifter in the reference arm to introduce a stepwise phase 


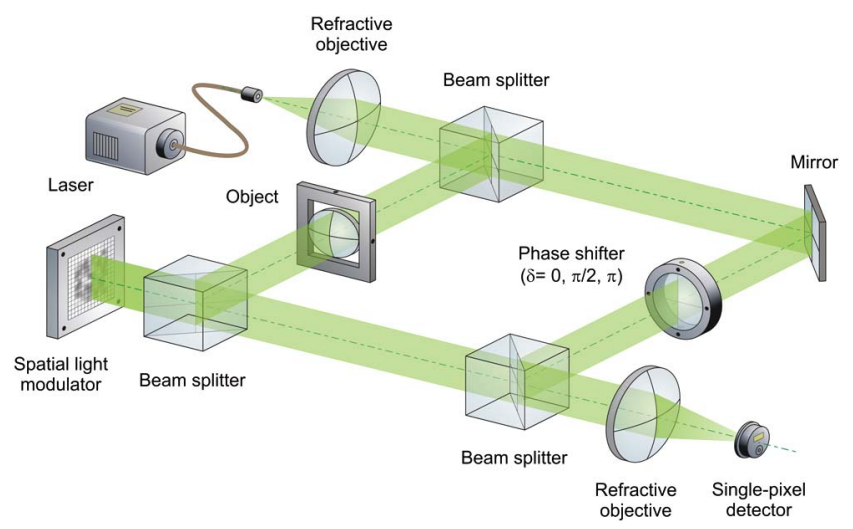

Fig. 1. Experimental setup for compressive phase-shifting holography. Light diffracted by the phase object is spatially modulated onto a SLM and, afterward, it interferes with the phase-delayed reference beam. The integrated energy flux of the signal is subsequently recorded with a single-pixel detector.

difference $\delta$. Our single-pixel phase-imaging holocamera employs a reflective 2D liquid crystal on silicon display (LCoS-SLM, Holoeye LC-R 2500) with XGA resolution and a pixel pitch of $19 \mu \mathrm{m}$ in the signal beam. The SLM generates a set of binary patterns to sample a fixed but otherwise arbitrary near-field diffraction pattern of the input phase object (an ophthalmic lens in our case).

For the practical implementation of the CS acquisition process, it is essential to determine the incoherent basis. A suitable choice corresponds to the Hadamard-Walsh functions. Hadamard matrices are square arrays of plus and minus ones, whose rows are orthogonal. Each row of a Hadamard matrix can be interpreted as a rectangular wave (Walsh function). Hadamard matrices perform the decomposition of a function by a set of rectangular waveforms, instead of the usual harmonic components associated with the Fourier transform. From an experimental point of view, the acquisition process using the Hadamard-Walsh basis is carried out by the generation of a collection of auxiliary binary $(0$ and +1$)$ intensity patterns easily imprinted onto the SLM. To record the intensity corresponding to a certain Hadamard-Walsh matrix $H$ we subtract the intensities generated from the binary patterns $H^{+}=(J+H) / 2$ and $H^{-}=(J-H) / 2$ codified onto the SLM, where $J$ is a matrix consisting of all $1 \mathrm{~s}$. This approach has the advantage of alleviating fluctuations in the light flux coming from the light source during hologram measurement. In what follows, we will refer to the sampling function $H$, although the above measurement process is indeed considered.

From elementary paraxial diffraction theory, when $H_{i}(\vec{r})$ is codified onto the SLM, the complex lightfield $U_{i}^{\text {signal }}(\vec{r})$ just after the SLM plane with transverse coordinate $\vec{r}$ in Fig. 1 is

$$
U_{i}^{\text {signal }}(\vec{r})=t(\vec{r} ; z) H_{i}(\vec{r}),
$$

where $t(\vec{r} ; z)$ is a short-hand notation for light distribution propagated a distance $z$ from the object to the SLM, i.e., $t(\vec{r} ; z)=t(\vec{r}) \otimes \exp \left(j k \vec{r}^{2} / 2 z\right)$, with $t(\vec{r})$ the phase object, $k$ the wavenumber, $j$ the imaginary unit, and $\otimes$ the symbol for the convolution operation.
To adapt the concept of single-pixel camera to phaseshifting interferometry we perform the following steps. First, a series of $M$ binary patterns $H_{i}(\vec{r})$ are sequentially displayed onto the SLM. For each pattern, we combine the diffracted field generated from $U_{i}^{\text {signal }}(\vec{r})$ with the phase-shifted reference beam $U_{\delta}^{\text {reference }}(\vec{r})$, which with no lack of generality is assumed to be a plane wave. In our case, a liquid crystal variable retarder is used as a phase shifter and three interference patterns (corresponding to a phase delay of $\delta=0, \delta=\pi / 2$, and $\delta=\pi$ ) are considered. As in conventional phase-shifting holography [2], the hologram is retrieved as

$$
\begin{aligned}
U_{i}^{\text {signal }}\left(\vec{r} ; z^{\prime}\right)= & \frac{1}{4}\left[I_{i, 0}(\vec{r})-I_{i, \pi}(\vec{r})\right] \\
& +\frac{j}{4}\left[2 I_{i, \pi / 2}(\vec{r})-I_{i, 0}(\vec{r})-I_{i, \pi}(\vec{r})\right],
\end{aligned}
$$

where

$$
I_{i, \delta}(\vec{r})=\left|U_{i}^{\text {signal }}\left(\vec{r} ; z^{\prime}\right)+U_{\delta}^{\text {reference }}\right|^{2}
$$

is the interferogram of the diffracted field from the SLM with the phase-shifted reference. Again the short-hand notation $U_{i}^{\text {signal }}\left(\vec{r} ; z^{\prime}\right)$ for light distribution propagated a distance $z^{\prime}$ from the SLM to the detector is employed.

Second, the hologram in Eq. (2) is integrated according to the single-pixel detection scheme proposed in Fig. 1 . The signal of interest is

$$
y_{i}=\iint U_{i}^{\text {signal }}\left(\vec{r} ; z^{\prime}\right) \mathrm{d}^{2} \vec{r}=\iint U_{i}^{\text {signal }}(\vec{r}) \mathrm{d}^{2} \vec{r},
$$

where the properties of the kernel of Fresnel propagation have been considered. Combining Eqs. (2) and (4) we find

$$
y_{i}=\frac{1}{4}\left[I_{i, 0}-I_{i, \pi}\right]+\frac{j}{4}\left[2 I_{i, \pi / 2}-I_{i, 0}-I_{i, \pi}\right],
$$

with

$$
I_{i, \delta}=\iint I_{i, \delta}(\vec{r}) \mathrm{d}^{2} \vec{r}
$$

In short, for each binary pattern $H_{i}(\vec{r})$ the integrated intensities of the three phase-shifted interferograms are recorded to get the measurement $y_{i}$.

Finally, taking into account Eqs. (4)-(6), and after sampling so that integrals are replaced by summations, the imaging process is expressed mathematically by the relation

$$
\mathbf{y}=\mathbf{H x},
$$

where $\mathbf{y}$ is the collection of measurements, $\mathbf{H}$ is the measurement matrix (a row-wise array of all projection modes defined through the Hadamard-Walsh functions), and $\mathbf{x}$ is the sampled object. The identity expressed in Eq. (4) is crucial for the correctness of the above expression, since the measurement matrix is formed by the Hadamard modes and the object corresponds to a sampled version 
of the propagated field $t(\vec{r} ; z)$. Although identical in form to the algebraic problem in single-pixel imaging based on CS [주의, Eq. (7) has a major different physical implication as $\mathbf{x}$ denotes the complex amplitude of the sample and not its intensity, which corresponds to the well-known classical compressive imaging problem. The phase information is retained as a main finding in our proposal.

CS allows reconstruction of the correct $\mathbf{x}$ although the number of measurements $M$ is lower than the number $N$ of pixels of the sampled phase object. Because $M<N$, the system is undetermined; a given $\mathbf{y}$ does not specify a unique $\mathbf{x}$. The correct $\mathbf{x}$ is recovered by solving the optimization problem []]

$$
\min \|\mathbf{x}\| \text {, such that } \mathbf{y}=\mathbf{H x} \text {, }
$$

where $\|\mathbf{x}\|$ is the $\ell_{1}$ norm of the object represented in an appropriate basis, which reflects the inherent sparsity that exists in natural objects. Given a sufficiently large number $M$, the problem is rigorously solvable and the recovered signal $\mathbf{x}$ approaches the exact signal with high probability. For a $k$-sparse signal, the required $M$ scales as $k \log (N / k)$.

Note that CS recovers the complex amplitude of the propagated field, $t(\vec{r} ; z)$. The final step is to find the phase profile $t(\vec{r})$ of the sample. This fact is accomplished by computing the discrete back propagated Fresnel integral for a distance $-z$. Alternatively, we use the propagating transfer function method [3]],

$$
t(m, n)=F^{-1}\left\{F[t(m, n ; z)] \exp \left[\frac{j \pi \lambda z}{N(\Delta x)^{2}}\left(u^{2}+v^{2}\right)\right]\right\},
$$

where $F$ denotes fast Fourier transform, $(u, v)$ are discrete spatial frequency variables, $(m, n)$ are discrete spatial transverse coordinates, and $N$ is the number of pixels of $\mathbf{x}$ (we assume the same number of pixels for both orthogonal coordinates). In this procedure we consider the same resolution for all propagation distances and $\Delta x$ represents the pixel size of the recovered vector $\mathbf{x}$.

As a proof-of-concept, the phase image of an ophthalmic lens, used to correct myopia, was retrieved by means of the setup shown in Fig. 1. A solid-state laser (Oxxius slim-532) emitting at $532 \mathrm{~nm}$ was employed as the light source. The lens was placed $253 \mathrm{~mm}$ in front of the SLM, which sequentially generated a set of $M$ HadamardWalsh functions of $N=128 \times 128$ pixels. The pixel size was $19 \mu \mathrm{m}$ and we select $M=0.2 N$, which provides a good compromise between SNR and computational effort. The programming code for the CS reconstruction was the function lleq-pd of the l1-magic software package, which solves the standard basis pursuit problem
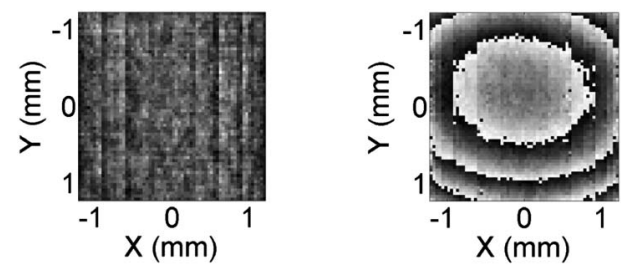

Fig. 2. Retrieved amplitude (left) and phase (right) of the field diffracted by the ophthalmic lens at the SLM plane.

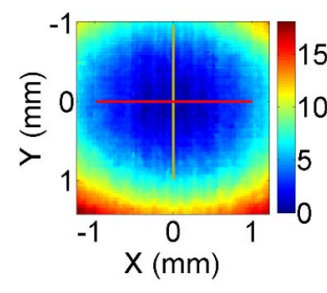

(a)

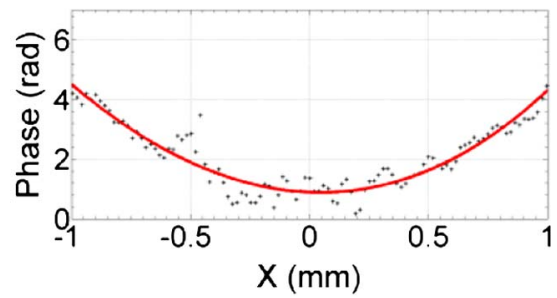

(c)

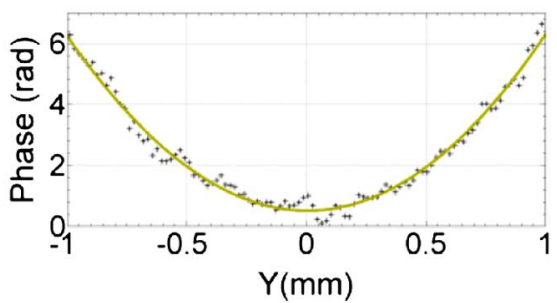

(d)

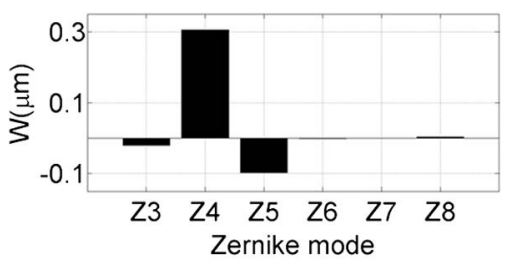

(b)
Fig. 3. (a) Phase map of the lens, (b) Zernike coefficients expansion, and fitted curves for the (c) horizontal and (d) vertical profiles marked in (a).

using a primal-dual algorithm [14]. The recovered amplitude and phase of the diffracted pattern at the SLM plane are plotted in Fig. 2. The phase image of the lens, retrieved by backward propagation of $t(\vec{r} ; z)$ after phase unwrapping, is shown in Fig. 3(a). This phase function was decomposed into the Zernike polynomial basis. The values (in microns) of the most representative Zernike coefficients are plotted in Fig. 3(b). From $Z_{4}$ (defocus) and $Z_{5}$ (astigmatism), the optical powers through the horizontal and vertical meridians of the lens were estimated to be $-1.11 \mathrm{dpt}$ and $-1.75 \mathrm{dpt}$, respectively, which is in good agreement with the specifications measured by a lens meter. To emphasize this astigmatic behavior, in Figs. 3(c) and $3(\mathrm{~d})$ we show the phase profiles along the horizontal and vertical axis marked in Fig. 3(a).

This research was funded by the Spanish Ministerio de Economía y Competitividad through project FIS201015746. Partial support from the Generalitat Valenciana and Universitat Jaume I through the projects PROMETEO/ 2012/021, ISIC/2012/013, and P1-1B2012-55 is also acknowledged.

\section{References}

1. M. Mir, B. Bhaduri, R. Wang, R. Zhu, and G. Popescu, Prog. Opt. 57, 133 (2012).

2. I. Yamaguchi and T. Zhang, Opt. Lett. 22, 1268 (1997).

3. L. Martínez-León, M. Araiza, B. Javidi, P. Andrés, V. Climent, J. Lancis, and E. Tajahuerce, Opt. Mater. Express 17, 12900 (2009). 
4. T. Kakue, R. Yonesaka, T. Tahara, Y. Awatsuji, K. Nishio, S. Ura, T. Kubota, and O. Matoba, Opt. Lett. 36, 4131 (2011).

5. I. Yamaguchi, K. Yamamoto, G. A. Mills, and M. Yokota, Appl. Opt. 45, 975 (2006).

6. D. L. Donoho, IEEE Trans. Inf. Theory 52, 1289 (2006).

7. E. Candes, J. Romberg, and T. Tao, IEEE Trans. Inf. Theory 52, 489 (2006).

8. M. F. Duarte, M. A. Davenport, D. Takhar, J. N. Laska, T. Sun, K. F. Kelly, and R. G. Baraniuk, IEEE Signal Process. Mag. 25, 83 (2008).
9. W. L. Chan, K. Charan, D. Takhar, K. F. Kelly, R. G. Baraniuk, and D. M. Mittleman, Appl. Phys. Lett. 93, 121105 (2008).

10. R. D. Swift, R. B. Wattson, J. A. Decker, R. Paganetti, and M. Harwit, Appl. Opt. 15, 1595 (1976).

11. D. J. Brady, K. Choi, D. L. Marks, R. Horisaki, and S. Lim, Opt. Mater. Express 17, 13040 (2009).

12. Y. Rivenson, A. Stern, and B. Javidi, Appl. Opt. 52, A423 (2013).

13. P. Clemente, V. Durán, E. Tajahuerce, V. Torres-Company, and J. Lancis, Phys. Rev. A 86, 041803(R) (2012).

14. E. Candes, http://www-stat.stanford.edu/ candes/11magic. 